\title{
An Updated Line List for NUV Spectral Synthesis in Evolved Stars: Redetermination of the Beryllium Abundance in the Solar Photosphere
}

\author{
Joleen K. Carlberg ${ }^{1}$ (i), Katia Cunha ${ }^{2,3}$, Verne V. Smith ${ }^{4}$, and José-Dias do Nascimento, Jr. ${ }^{5,6}$ \\ ${ }^{1}$ Space Telescope Science Institute, 3700 San Martin Drive, Baltimore, MD 21218, USA; jcarlberg@stsci.edu \\ 2 Observatório Nacional, Rua General José Cristino, 77, 20921-400 São Cristóvão, Rio de Janeiro, RJ, Brazil \\ Steward Observatory, University of Arizona, Tucson, AZ 85719, USA \\ ${ }^{4}$ National Optical Astronomy Observatory, 950 North Cherry Avenue, Tucson, AZ 85719, USA \\ ${ }^{5}$ Departamento de Física, Universidade Federal do Rio Grande do Norte, 59072-970 Natal, RN, Brazil \\ ${ }^{6}$ Harvard-Smithsonian Center for Astrophysics, 60 Garden Street, Cambridge, MA 02138, USA \\ Received 2017 December 30; revised 2018 July 29; accepted 2018 July 30; published 2018 September 17
}

\begin{abstract}
Motivated by the desire to measure beryllium abundances in red giant stars, we have constructed a new line list for synthesizing spectra in the near-ultraviolet. Using the Sun, Arcturus, and Pollux as benchmark stars, we explore potential sources of missing continuous opacity and line absorption. Despite the inclusion of new sources of continuous opacity, fitting the ultraviolet $\mathrm{OH}$ lines in the solar spectrum still requires artificially inflating the continuous opacity. The red giants also require the addition of a line of unknown origin in the blue wing of the Be line at $\lambda 3131.065 \AA$, and we find a good match can be made by adopting a Ti II line with low excitation potential. The inclusion of this line does not affect the measurement of the solar Be abundance. Because the fits to all of the benchmark stars require tuning the properties of known spectral lines and including both an unknown source of additional continuous opacity and spectral lines of unknown origin, we argue that the absolute abundances of Be in stars should be interpreted with caution. Therefore, the question of whether the solar Be abundance is depleted may not yet be resolved, although our model favors minimal Be depletion in the Sun.
\end{abstract}

Key words: stars: abundances - stars: atmospheres - stars: late-type - Sun: abundances

Supporting material: machine-readable table

\section{Introduction}

Beryllium is a trace element in stellar photospheres and is one of a few fragile elements (like $\mathrm{Li}$ and $\mathrm{B}$ ) that are destroyed in the stellar interior. The temperature at which Be is efficiently destroyed is intermediate to that of $\mathrm{Li}$ (which shows a wellknown depletion in the solar photosphere compared to meteoritic measurements) and B (which is undepleted in the solar photosphere; Cunha \& Smith 1999). These three elements can be used together to constrain the depth of mixing in stars with outer convection zones. Unfortunately, there are few absorption lines in stellar spectra with which the $\mathrm{Be}$ abundances can be measured. The most commonly used lines are the two Be II lines in the near-ultraviolet (NUV) around $\sim 3130 \AA$ A just barely within the wavelength cut-off at which the Earth's atmosphere becomes opaque to incoming radiation. Cool stars, i.e., those with convective envelopes, are relatively faint at these wavelengths, where these stars' spectral energy distributions are falling steeply toward shorter wavelengths.

As is often the case, the Sun is the star that has the beststudied Be abundance. The amount by which Be has been depleted in the solar photosphere can be found by comparing its relative abundance to that measured from $\mathrm{Cl}$ chondritic meteorites, which preserve the primordial abundance pattern of the early solar system. Earlier meteoritic abundance measurements yielded $A(\mathrm{Be})=1.42 \mathrm{dex}^{7}$ (Grevesse \& Sauval 1998), while more recent measurements yield an average of 1.32 dex (Lodders 2010). Historically, it was generally accepted that the solar photosphere was depleted in Be (Chmielewski et al. 1975) by a factor of about $1.7(A(\mathrm{Be})=1.15 \mathrm{dex})$, until

\footnotetext{
7 The number abundance of an element, $\mathrm{X}$, in this notation is defined as $A(\mathrm{X}) \equiv \log N_{\mathrm{X}}-\log N_{\mathrm{H}}+12$.
}

Balachandran \& Bell (1998) demonstrated that NUV OH lines in one-dimensional (1D) synthetic spectra were deeper than what was seen in the observations. They interpreted this discrepancy to be the result of a missing source of continuous opacity. This missing opacity had also been put forth to resolve discrepancies between observed and predicted values of the solar irradiance (e.g., Kurucz 1992) and stellar UV colors (e.g., Bell et al. 1994). A larger opacity creates shallower model Be lines for a fixed abundance, meaning that the Be abundance had to be increased from previous determinations to match observations. This result was later confirmed with a threedimensional (3D) analysis by Asplund (2004). Consequently, an undepeleted photospheric $A(\mathrm{Be})$ became the prevailing wisdom (e.g., the compilation of photospheric abundances by Asplund et al. (2009) adopts $1.38 \mathrm{dex}$ ), and a search for the source of the Sun's "missing opacity" ensued (Bell et al. 2001; Castelli \& Kurucz 2004; Short \& Hauschildt 2005, 2009). However, the interpretation of Balachandran \& Bell (1998) relies on an a priori knowledge of the solar $A(\mathrm{O})$, and is complicated by the fact that the measurement of the solar oxygen abundance has yielded steadily decreasing values as stellar atmosphere modeling has improved. Other groups contest this reduction in the oxygen abundance, and this controversy led Lodders (2003) to adopt the older, depleted $A$ (Be) of $1.15 \pm 0.20$ in her compilation of solar photospheric abundances. Additional analyses of the Sun have also argued in favor of the depleted value, such as Boesgaard \& King (2002) who find $A(\mathrm{Be})=1.15 \mathrm{dex}$ for the Sun in their paper on dwarfs in the Hyades. They find meteoritic $A(\mathrm{Be})$ of Hyades dwarfs with similar temperatures as the Sun, arguing against any systematic errors in their analysis and supporting the idea that the Sun's Be is truly depleted. 
Table 1

Adopted Stellar Abundances

\begin{tabular}{|c|c|c|c|c|c|c|}
\hline Element & $\begin{array}{l}A(\mathrm{X}) \\
(\mathrm{dex})\end{array}$ & References & $\begin{array}{l}A(\mathrm{X}) \\
(\mathrm{dex})\end{array}$ & References & $\begin{array}{l}A(\mathrm{X}) \\
(\mathrm{dex})\end{array}$ & References \\
\hline & \multicolumn{2}{|c|}{ Sun } & \multicolumn{2}{|c|}{ Arcturus } & \multicolumn{2}{|c|}{ Pollux } \\
\hline $\mathrm{Li}$ & 1.22 & A09 & $<-0.73$ & $\mathrm{C} 12$ & +0.72 & L07 \\
\hline $\mathrm{N}$ & 8.66 & A09 & 8.10 & R11 & 8.66 & L07 \\
\hline $\mathrm{O}$ & 8.93 & A09 & 8.67 & R11 & 8.93 & L07 \\
\hline $\mathrm{Mg}$ & 7.82 & A09 & 7.45 & $\mathrm{R} 11$ & 7.82 & L07 \\
\hline $\mathrm{Mn}$ & 5.70 & A09 & 4.70 & R11 & 5.70 & L07 \\
\hline $\mathrm{Fe}$ & 7.62 & A09 & 7.10 & R11 & 7.79 & L07 \\
\hline $\mathrm{Zr}$ & 2.75 & A09 & $\ldots$ & & $\ldots$ & \\
\hline
\end{tabular}

References. A09: Asplund et al. (2009); C12: Carlberg et al. (2012); R11: Ramírez \& Allende Prieto (2011); L07: Luck \& Heiter (2007).

The continuous opacity is not the only aspect of the spectral synthesis modeling that is incomplete. There is no single agreed upon line list for generating model spectra, and the more isolated of the two Be II lines is blended with another line of disputed origin. A known transition of Mn I exists at the correct wavelength ( $3131.037 \AA$ ) but is too weak to account for the observed profile. Different groups solve this discrepancy either by increasing the $\log g f$ of this known transition or introducing an additional "hypothetical" iron line.

Although such line list tuning should work well for stars with similar properties to the Sun, uncertainties may be much larger in stars of different properties. We are ultimately interested in measuring $A(\mathrm{Be})$ in a sample of red giant stars (J. K. Carlberg et al. 2018, in preparation), necessitating a close look at the effects of the various line-tuning schemes on the $A$ (Be) of red giants. For this work, we select three benchmark stars (the Sun and two well-studied red giants for which we can make assumptions about their $A(\mathrm{Be})$, Section 2) with which to test synthetic spectral models that utilize the most recently identified sources of continuous opacity (Section 3) and to test the validity of different line lists across a wide range of $T_{\text {eff }}$ and $\log g$ (Section 4). We find that the solar spectrum still requires the addition of extra continuous opacity over what is present in the models (Section 3) and that the red giants' spectra suggest an alternative line-solution tuning than those used previously in the literature (Section 4). We use these new models to re-analyze the solar photospheric $A(\mathrm{Be})$ (Section 5) and summarize the limitations still inherent in $A(\mathrm{Be})$ measurements (Section 6).

\section{The Benchmark Stars: Properties and Spectral Data}

The Sun is the most well-characterized star and provides a benchmark for nearly all stellar studies. We adopt the MARCS (Gustafsson et al. 2008) standard solar atmospheric model, which has parameters of $T_{\text {eff }}=5777 \mathrm{~K}, \log g=4.44$, and $\xi=1.00 \mathrm{~km} \mathrm{~s}^{-1}$. This atmosphere model was calculated using the Grevesse et al. (2007) solar composition, whereas the default solar composition adopted in this work is that of Asplund et al. (2009). The latter has slightly higher abundances (0.03-0.07 dex) of some of the most abundant metals in the Sun (e.g., C, N, O, Mg, and Fe). In Table 1, we list the adopted solar abundances for key elements affecting the spectral synthesis.
The Sun is obviously not a suitable representative of red giant branch stars. One of the most well-characterized red giants is Arcturus, with high-quality spectral atlases available (Hinkle et al. 2000, 2005), detailed abundance analysis (e.g., Smith et al. 2000 and Ramírez \& Allende Prieto 2011), and interferometric angular diameter measurements (e.g., Lacour et al. 2008 and Richichi et al. 2009). Arcturus has marked nonsolar ratios of $[\mathrm{X} / \mathrm{Fe}]$ for many elements with significant contributions to the opacities and line formation in the NUV. We adopt both the stellar atmospheric parameters $\left(T_{\text {eff }}=4286\right.$ $\mathrm{K}, \log g=1.66,[\mathrm{Fe} / \mathrm{H}]=-0.52$, and $\xi=1.74 \mathrm{~km} \mathrm{~s}^{-1}$ ) and elemental abundances from Ramírez \& Allende Prieto (2011). The MARCS atmosphere model for Arcturus ( $\alpha$ Boo) is interpolated from the $\alpha$-enhanced grid. The MARCS database of models also offers CN-processed models, but does not provide models that have both $\alpha$-enhancement and $\mathrm{CN}$ processing. The differences in the input chemistry of the models will alter the physical properties of the model, but this only has a small effect on the resulting model spectrum since the gross properties of the model spectrum are more heavily influenced by the abundances adopted to calculate the line absorption.

Many red giant stars have global properties that are intermediate to the Sun and Arcturus; therefore, we also test our line list on the bright red giant, Pollux $(\beta \mathrm{Gem})$, which is well-characterized though not to the extent of Arcturus. To model Pollux, we adopted stellar parameters from Luck \& Heiter (2007) $\left(T_{\text {eff }}=4998 \mathrm{~K}\right.$, $\left.\log g=3.13 \mathrm{dex},[\mathrm{Fe} / \mathrm{H}]=+0.17 \mathrm{dex}, \xi=1.47 \mathrm{~km} \mathrm{~s}^{-1}\right)$. Individual abundances for the 24 elements available in Luck \& Heiter (2007) were explicitly set in the spectral synthesis calculations, while the remaining abundances assume scaled solar values.

All of the observational data used in this work are archival spectra. The solar spectrum is a Subaru HDS observation of the asteroid Vesta, previously analyzed by Takeda et al. (2011). The raw data and associated calibration files were downloaded from the Subaru Mitaka Okayama Kiso Archive (SMOKA; Baba et al. 2002). Details of the observations are given in Table 2. The data were reduced with IRAF, following the HDS Data Reduction Guide. ${ }^{8}$ We used facility provided scripts for the overscan correction and linearity correction (Tajitsu et al. 2010.). The images were corrected for bias, flat-fielding, bad pixels, and cosmic rays. Scattered light was removed

\footnotetext{
8 Available at http://www.subarutelescope.org/Observing/Instruments/HDS/ specana2014.10e.pdf.
} 
Table 2

Observation Log

\begin{tabular}{lccccc}
\hline \hline Star & Telescope & Instrument & UT Date & $N$ & $\begin{array}{r}T_{\text {exp }} \\
(\mathrm{s})\end{array}$ \\
\hline Arcturus & $H S T$ & STIS & 2003 Mar 01 & 2 & 3315 \\
Pollux & $H S T$ & STIS & 2014 May 28 & 1 & 1809 \\
Vesta & Subaru & HDS & 2010 Feb 05 & 3 & 600 \\
\hline
\end{tabular}

before extracting the spectra. Wavelength solutions were applied from Th-Ar lamp spectra taken nearest in time to each stellar spectrum. A tenth-order Legendre polynomial was fit to the apparent continuum in each echelle order to measure and remove the blaze function. The overall continuum was multiplied by a factor of 0.78 to obtain a good match between the observed and synthetic spectra. The instrumental broadening was measured from the comparison lamp spectra and was found to be $0.05 \AA$ (FWHM); however, to get a good fit to the more isolated atomic lines, we found that we had to increase the instrumental broadening to $0.075 \AA$. This empirical broadening was used for all solar syntheses.

The red giant spectra come from the StarCAT (Ayres 2010) and ASTRAL (Ayres 2013) catalogs, which provides fluxcalibrated spectral coverage of a large swath of the FUV/NUV spectrum "stitched" together from multiple observing configurations of the Space Telescope Imaging Spectrograph (STIS) on the Hubble Space Telescope (HST). The spectral coverage used here is provided by the echelle E320H setting centered at $3012 \AA$, and the observing times are given in Table 2 . For the red giants, we modeled all sources of broadening with a Gaussian profile of widths 0.08 and $0.065 \AA$, for Arcturus and Pollux, respectively. Because the red giant spectra are flux calibrated, we normalized the spectrum by dividing by flux models calculated using the 2014 version of the stellar synthesis code MOOG $^{9}$ (Sneden 1973). The model fluxes were scaled by $(R / d)^{2}$, using the Hipparcos parallaxes to obtain distance $(d)$ and adopting $R=8.8 R_{\odot}$ (Hatzes et al. 2006) for Pollux and $R=25.4 R_{\odot}$ (Ramírez \& Allende Prieto 2011) for Arcturus. This normalization results in an overall satisfactory agreement with the synthetic models and, in the case of Arcturus, is in qualitative agreement with the overall normalization of the spectrum presented in Melo et al. (2005). Therefore, no additional adjustment to the overall scaling of the spectra are made.

\section{The UV Continuous Opacity}

There has been some question on the completeness of the continuous opacity calculations and the effects of the missing contributions to the measurement of $A(\mathrm{Be})$ in the solar spectrum. Underestimating the opacity will lead to incorrect tuning of lines with uncertain $\log g f$ values and predicting too deep lines when the $\log g f$ is well constrained. As a consequence, abundance measurements will systematically underestimate the true elemental abundances.

As previously mentioned in Section 1, Balachandran \& Bell (1998) argued this case for the Sun based on modeling a set of $\mathrm{OH}$ lines with well-known $g f$-values. They required increasing the total opacity by a factor of 1.6 to reproduce the lines using

\footnotetext{
9 Available at http://www.as.utexas.edu/ chris/moog.html.
}

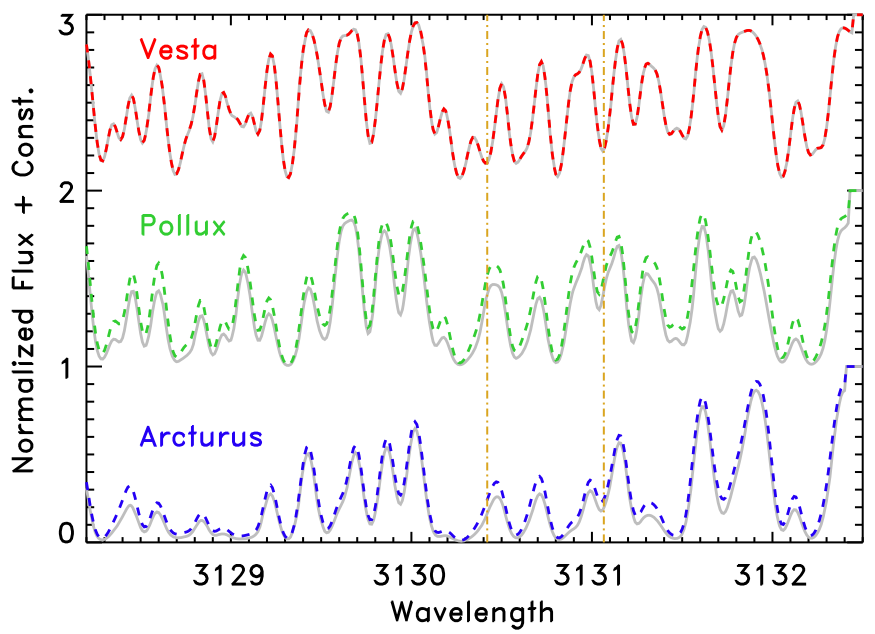

Figure 1. Comparison of synthetic spectra generated by the 2014 version of MOOG (solid gray lines) and those generated by our modified version of the code that includes opacity from the dissociation of $\mathrm{CH}$ and $\mathrm{NH}$ (dashed lines), keeping all other inputs the same. In the solar spectrum (top), the additional opacity has a negligible effect on the emergent spectrum. For red giants Pollux and Arcturus, the effect is more substantial, including in the vicinity of the Be II lines (vertical dashed-dotted lines).

the $\mathrm{O}$ abundance derived from infrared lines. However, Allende Prieto \& Lambert (2000) used UV fluxes from IUE to measure the effective temperature and metallicity of late-type stars and find good agreement with measurements using other methods. They find no missing opacity problem for the Sun or other latetype stars, though it is worth noting that their longest wavelength $(3000 \AA)$ does not quite reach the Be II lines. More recently, Smiljanic et al. (2009) argued that one can account for the "missing opacity" by increasing the adopted $\mathrm{Fe}$ abundances in the opacity calculations; however, for their metal-poor stellar sample, they found that changing the opacity had a negligible effect on their derived $A(\mathrm{Be})$.

Fontenla et al. (2015) recently presented an analysis of opacity from the dissociation of molecules, such as $\mathrm{CH}, \mathrm{NH}$, and $\mathrm{OH}$, in an effort to better reproduce the solar irradiance spectrum, and indeed, they could compute a model irradiance spectrum that matched the observed solar spectrum significantly better than when molecular dissociation was ignored. The effect is largest below $\sim 2000 \AA$, but both $\mathrm{CH}$ and $\mathrm{NH}$ present secondary peaks in opacity at $\sim 3000-4000 \AA$. We tested whether including this opacity would affect the continuum enough to alter the derived $A(\mathrm{Be})$ by modifying the source code of the 2014 version of MOOG to include dissociation of $\mathrm{CH}$ and $\mathrm{NH}$. The $\mathrm{CH}$ cross-sections come from Kurucz et al. (1987), while the NH data come from P. Stancil (private communication). We ran the spectral synthesis for the Sun, Arcturus, and Pollux using the literature line list available in Takeda et al. (2011) and found that the contribution by each molecule on the total opacity was no more than $\sim 4 \%$. As shown in Figure 1, there is little change to the computed spectrum for a Sun-like star, but a significant change for a red giant spectrum. Therefore, we use this modified version of MOOG to compute the spectra. The new and modified subroutines as well as the MOOG-formatted version of the line list presented in the next section are all available online ${ }^{10}$ (Carlberg 2018).

\footnotetext{
${ }^{10}$ Codebase: https://github.com/jkcarlberg/Be-synthesis-with-moog.
} 


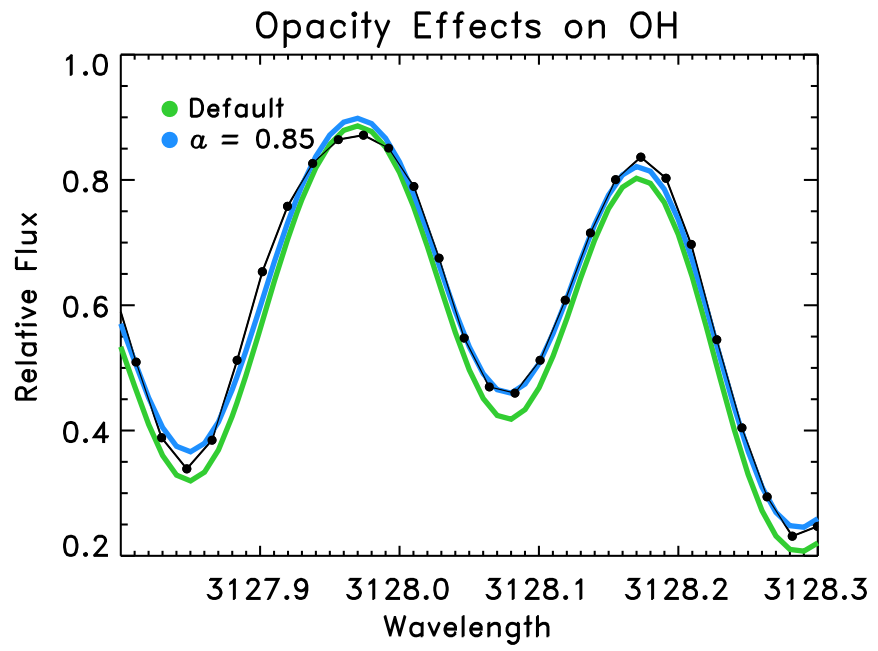

Figure 2. Synthesis of the solar $\mathrm{OH}$ line at $3128.060 \AA$ using the opacity calculations of MOOG plus the new $\mathrm{CH}$ and $\mathrm{OH}$ cross-sections (green line). Increasing the total opacity by an empirical factor of $0.85\left(10000 / T_{\tau}\right)$ significantly improves the fit (blue line).

Next, we tested whether the opacity was well modeled by synthesizing the $\mathrm{OH}$ feature at $3128.060 \AA$, using the line list we describe more fully in the next section. The results are show in Figure 2. We find, as did Balachandran \& Bell (1998), that the syntheses predict lines that are too deep even when including the new dissociation opacities and despite the fact that our adopted oxygen abundance is lower than that adopted in Balachandran \& Bell (1998). MOOG allows an artificial enhancement of the calculated continuous opacity by a factor of $a 10000 / T_{\tau}$, where $a$ is a positive number and $T_{\tau}$ is the temperature of the atmosphere layer. We tested a range of $a$ values from 0.1 to 1.2 and found that $a=0.85$ produces an excellent fit (Figure 2). At the mean depth of line formation for this line, $a=0.85$ corresponds to a factor of $\sim 1.2$ increase in the opacity. We explore the effects of the adopted oxygen abundance and inflated opacities on the derived $A(\mathrm{Be})$ in more detail in Section 5.

\section{Constructing a New Line List}

\subsection{Literature Values}

To take advantage of new line data recently made available, we have compiled a line list from scratch from a variety of sources. Molecular transitions for $\mathrm{CH}$ come from Masseron et al. (2014), while CN transitions in our list come from Sneden et al. (2014). Neutral iron lines were retrieved from Kurucz's website $^{11}$ plus newly identified lines from Peterson \& Kurucz (2015).

The remaining atomic and molecular species were selected by cross-referencing two recently published lists from Takeda et al. (2011, hereafter, T11) and Delgado Mena et al. (2012, hereafter, DM12). The DM12 list is larger and thus contributes the majority of lines and line parameters. A subset of lines common to both lists had large differences in their $\log g f$-values (by more than $0.1 \mathrm{dex}$ ). For these lines, we cross-referenced with the Vienna Atomic Line Database ${ }^{12}$ (Kupka et al. 1999) and adopted those line parameters. The one

\footnotetext{
11 gf2600.lines (file last modified 2016 June 05) available at http://kurucz. harvard.edu/atoms/2600/.

12 http://vald.astro.uu.se, accessed on 2016 August 02.
}

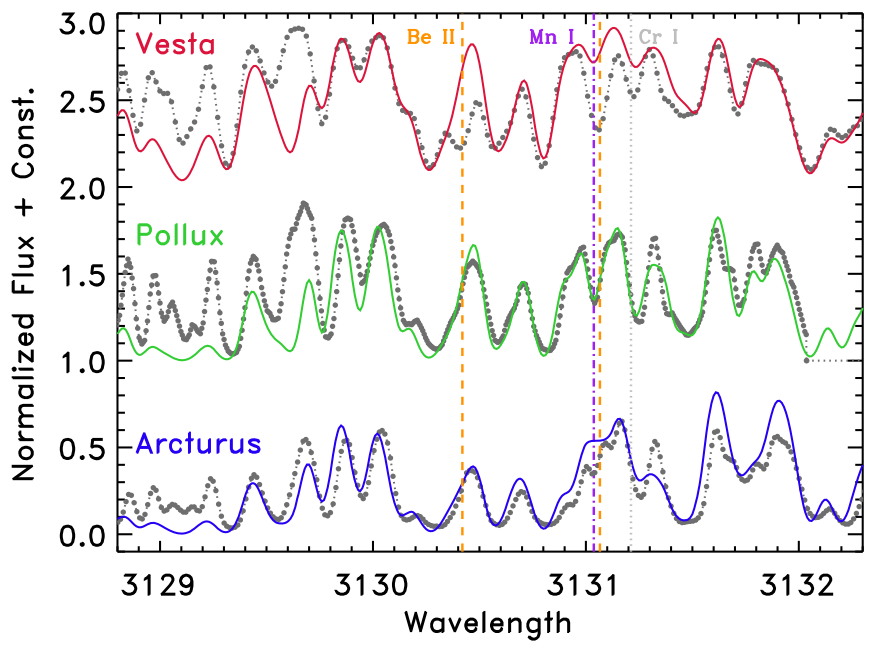

Figure 3. Comparison of synthetic spectra generated with the newly compiled line list (solid lines) compared to observations (dotted lines), assuming no Be in the stellar atmospheres. The dashed vertical lines show the location of the Be II lines, and the dotted-dashed vertical line marks the Mn I line at $\lambda 3131.037 \AA$, using the tuned $\log g f$ of -0.224 from DM12. The solar spectrum (top, archival Subaru spectrum of Vesta) is clearly not well fit when Be is absent from the model. The spectrum of Pollux (middle) shows a generally good fit at wavelengths above $\sim 3130.5 \AA$, whereas the model for Arcturus (bottom) has conspicuous missing absorption just blue ward of the $\lambda 3131.065 \AA$ Be II line.

exception is the discrepant $\log g f$ of the $\lambda 3131.037 \AA$ Mn I line. There is a contentious blend in the blue wing of the $\lambda 3131.066$ Be II line that has been modeled with either the known $\lambda 3131.037 \AA$ Mn I line, with its $\log g f$ tuned to match the solar spectrum (requiring a large, $\sim 1.5 \mathrm{dex}$, increase) or an unidentified Fe I line (see, e.g., Takeda et al. 2011 for hypothetical line properties.) In our initial line list, we use the former model, adopting the tuned $\log g f$ of -0.224 from DM12. This blended feature is addressed more fully in the next section.

We use this initial line list to generate synthetic spectra of the benchmark stars without any Be to compare to the observed spectra, as shown in Figure 3. The solar spectrum clearly requires the inclusion of $\mathrm{Be}$ absorption to fit the observed spectrum at the location of both Be II lines. In the modeled solar spectrum, the contentious Mn I line at $\lambda 3131.037 \AA$ is clearly visible in the blue wing of the Be line at $\lambda 3131.065 \AA$. The Mn I line is stronger in the red giant spectra and is well fit with the larger $\log g f$ adopted from DM12. All three benchmark observations show excess absorption predicted by the models, especially below $\lambda 3130.0 \AA$. Most concerning is that the Arcturus model is a poor fit to the spectrum in the contentious blue wing of the $\lambda 3131.065 \AA \mathrm{Be}$ line. Some tuning of the $g f$-values is therefore required.

\subsection{Tuning the Line List}

The poor fit seen at $\lambda \lesssim 3129.6 \AA$ has little effect on the Be abundance analysis, but we did trace the problem to two $\mathrm{Fe}$ lines: $\lambda 3129.102 \AA$ (Peterson \& Kurucz 2015) and $\lambda 3129.619$ (VALD K14). Removing these lines (which we achieved by changing the $\log g f$ by the arbitrarily large factor of -10 ) produces a much better fit to the spectrum. The focus of the remainder of astrophysical tuning is on a narrow wavelength region around the redder $\mathrm{Be}$ II line, which is the more isolated of the two lines. Despite the relative isolation, there are still significant blends including the contentious Mn I noted earlier 
as well as a contribution in the red wing of the line by $\mathrm{ZrI}$ at $\lambda 3131.109 \AA$. In the solar spectrum, this line is weak $(\sim 1.5 \mathrm{~m} \AA$ equivalent width), but the equivalent width is at least an order of magnitude larger in red giant stars. Furthermore, Figure 3 shows that a nearby $\mathrm{Cr} \mathrm{I}$ line is poorly fit in the solar spectrum and is nearby enough to potentially affect the neighboring $\mathrm{Be}$ blend. The synthesized Cr I line at $\lambda 3131.212 \AA$ is too weak in the spectra of all three benchmark stars. Its $\log g f$ was increased by 0.60 dex in order to match the observed solar spectrum.

As we considered additional changes to the line list in the blended Be feature, we generated spectra for both Arcturus and Pollux assuming no contribution from Be. This assumption is valid for Arcturus because Arcturus is heavily depleted in $\mathrm{Li}$ $(<-0.73$ dex, Carlberg et al. 2012); thus, it is reasonable to assume that $\mathrm{Be}$ is also heavily depleted. Pollux has depleted $A$ (Li), $\sim 1 / 3$ the solar photosphere value, but it is $\sim 30$ times larger than Arcturus. $A(\mathrm{Be})$ in Pollux should also be depleted, though it is not clear by how much. Beryllium may be contributing to the absorption in the observed Pollux spectrum. Therefore, while we can confidently exclude changes to the line list that result in model spectra with absorption exceeding what is seen in the observed Pollux spectrum, we do allow changes in the line list that result in less absorption in this feature since Be may be contributing.

Figure 4 shows the region of focus for the red giant spectra, where the blue model spectrum shows the line list with all the tuning made to the line list so far and is labeled "Mn I as in DM12" because it adopts the tuned $\log g f$ of Mn I used in that work. Although this line list provides a good fit to Pollux, there is significant missing absorption in the models for the Arcturus spectrum. We next tried fitting the region using the untuned $\mathrm{MnI}$ line and including the hypothetical Fe I line used by T11 at $\lambda 3131.039 \AA$ (purple line Figure 4). However, this resulted in too much absorption in the Pollux spectrum. Similarly, increasing the $\log g f$ of the $\mathrm{Zr}$ I line at $\lambda 3131.109 \AA$ produced too much absorption in Pollux (not shown). Furthermore, its absorption was too red to reproduce the missing absorption in Arcturus. Because the Be blend in Pollux's spectrum in Figure 4 with the tuned Mn I line is already well fit, we concluded that the missing absorption in Arcturus's spectrum must be from a species that is strong only in Arcturus. Thus, we speculate that it must be an ionized metal species (which become stronger in low $\log g$ stars) and that it must be an element that is relatively overabundant in Arcturus, i.e., $[\mathrm{X} / \mathrm{Fe}]>0$. We thus tried inserting a Ti II line at $\lambda 3131.039 \AA$, adopting the same excitation potential as the Ti II line at $\lambda 3130.803 \AA$ and adjusting the $\log g f$ to -4.400 dex to get a reasonable match to Arcturus. We also slightly reduced the $\log g f$ of the $\mathrm{ZrI}$ line at $\lambda 3131.109 \AA$. These changes result in the orange spectrum in Figure 4. As expected, this Ti II line has a much smaller effect on Pollux's spectrum than does the Fe I line that T11 utilized. This line list (labeled "Mn I/Ti II*/Zr I Tuned", where the asterisk marks the hypothetical line) is the one we adopt. In summary, it adopts astrophysically tuned $\log g f$ for $\mathrm{Mn}$ I, Zr I, and Cr I and includes a hypothetical Ti II line. In Table 3, we present the line list adopted in this work. Line parameters that have been tuned are noted in the last column.
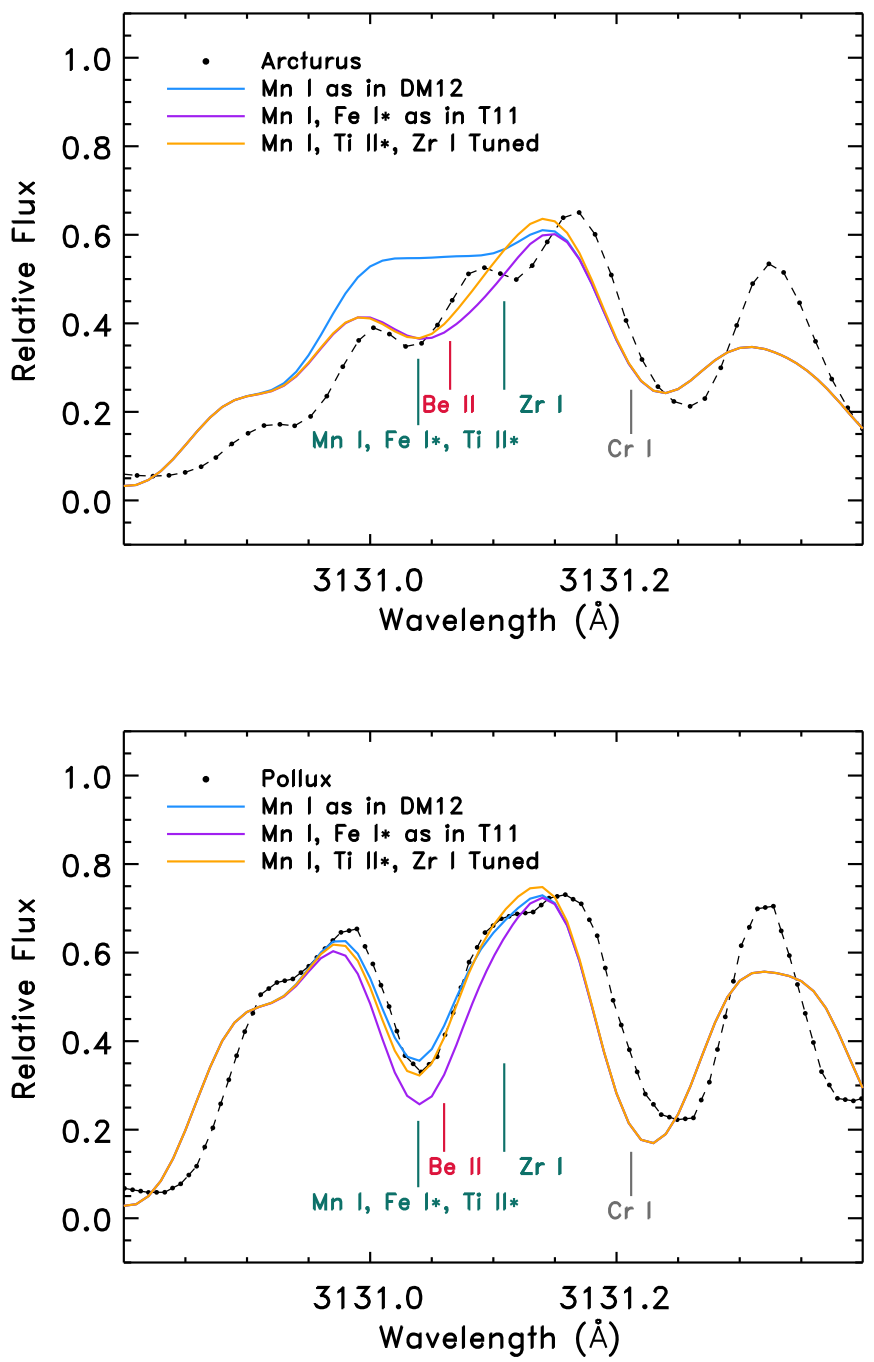

Figure 4. Observed spectrum of Arcturus (dashed line, top plot) and Pollux (dashed line, bottom plot) compared to synthetic spectra generated with three different line lists: Mn I with a tuned $\log g f$ (blue), Mn I with a laboratory $\log g f$ and a hypothetical Fe I line (purple), and the adopted line list which uses a hypothetical Ti II line and tuned $\log g f$ values for the Mn I and Zr I lines in the blend. The location of the Be II line (red) and tuned lines that are blended ( $\mathrm{Mn}$ and $\mathrm{Zr}$, teal) or neighboring ( $\mathrm{Cr}$, gray) the $\mathrm{Be}$ II line are also marked.

Table 3

Line List for Synthesizing Spectra

\begin{tabular}{lcccc}
\hline \hline $\begin{array}{l}\text { Wavelength } \\
\text { A }\end{array}$ & Species & $\begin{array}{l}\text { E.P. } \\
(\mathrm{eV})\end{array}$ & $\begin{array}{c}\log g f \\
\mathrm{dex}\end{array}$ & Source \\
\hline 3127.608 & ${ }^{12} \mathrm{CN}$ & 0.519 & -4.030 & S14 \\
3127.6096 & $\mathrm{Co} \mathrm{I}$ & 2.1367 & -5.851 & VALD \\
3127.6159 & $\mathrm{Ni} \mathrm{II}$ & 8.4162 & -5.187 & VALD \\
3127.6162 & $\mathrm{Ni} \mathrm{I}$ & 3.3062 & -3.830 & VALD \\
3127.6241 & $\mathrm{Cr} \mathrm{I}$ & 2.7079 & -3.991 & VALD \\
3127.6414 & ${ }^{12} \mathrm{CH}$ & 0.051 & -1.520 & M14 \\
3127.6543 & $\mathrm{Ni} \mathrm{I}$ & 3.3062 & -1.531 & VALD \\
\hline
\end{tabular}

(This table is available in its entirety in machine-readable form.)

\subsection{NLTE Effects}

All of these analysis have been calculated under localthermodynamic equilibrium (LTE) conditions even though departures from LTE are anticipated. The largest effects should be the NLTE corrections affecting the Be lines themselves as 


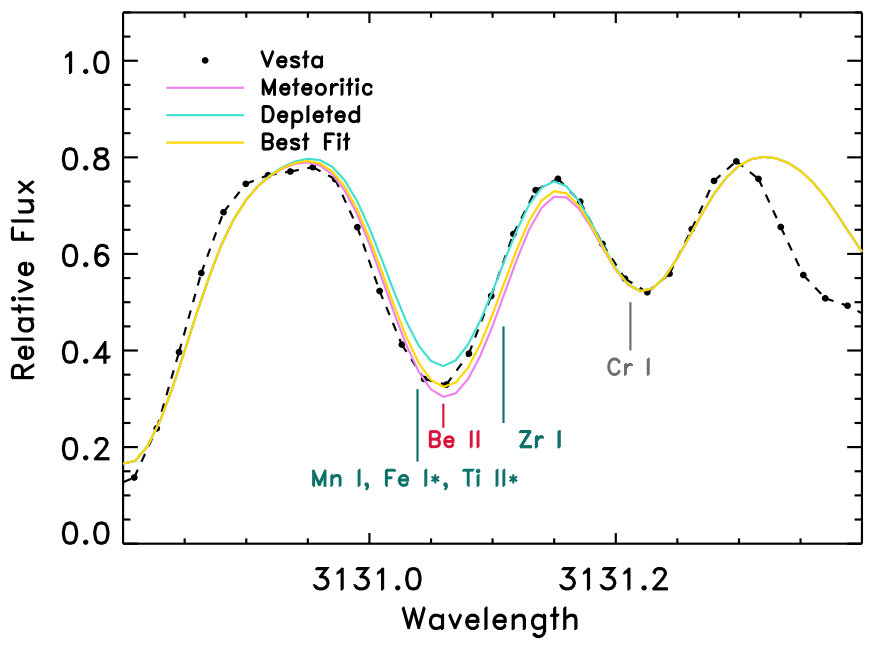

Figure 5. Solar spectrum from an observation of Vesta (dashed line) compared to synthetic spectra generated with three different beryllium abundances: meteoritic $(A(\mathrm{Be})=1.38 \mathrm{dex})$, depleted $(A(\mathrm{Be})=1.15 \mathrm{dex})$, and our best fit $(A(B e)=1.30$ dex $)$. Lines that have been tuned in our analysis are labeled as in Figure 4.

well as any lines that are blended with them. Given our focus on the line of Be II at $\lambda 3131.065 \AA$, the elements that are most relevant to consider for NLTE effects are Be, Mn, Ti and Fe. In solar-type stars, two competing NLTE effects (overionization and overexcitation) effectively cancel (Chmielewski et al. 1975), rendering the need for a correction unnecessary. Garcia Lopez et al. (1995) studied the effect on a wider range of metallicities and found the overall correction still remains small ( $\lesssim 0.1$ dex). Their sample included both dwarfs and stars near the base of the red giant branch, with $\log g \sim 3.5$. The correction for Mn is similarly small. In a study by Bergemann \& Gehren (2008), the corrections for stars with near-solar metallicity are again $\lesssim 0.1$ dex. Their study focused on dwarfs and subgiants, but they tested the effects of surface gravity down to $\log g=3$ and found that gravity only alters the results at low metallicity $(<-2$ dex) or high effective temperature $(>6000 \mathrm{~K})$. Therefore, the red giants should also not be affected. Asplund (2005) reviewed the state of the literature for NLTE corrections of a wide range of elements, and for the elements $\mathrm{Ti}$ and $\mathrm{Fe}$, there is no consensus on how significant the LTE departures are or how they vary with stellar parameters. However, it is reasonably well understood that NLTE corrections for Fe are likely small for solar-type stars and for solar metallicity stars. In light of these studies, we restrict our analysis to LTE.

\section{The Solar Be Abundance}

Utilizing the new opacity models and the updated and tuned line list described in the previous sections, we re-derive the solar photospheric abundance of $\mathrm{Be}$, focusing on the more isolated line at $3131.065 \AA$. The best fit is found by running MOOG interactively and adjusting only the abundance of Be to minimize the difference between the observed and generated spectra in the very narrow wavelength region encompassing the blended feature (3130.95-3131.15 $\mathrm{A}$ ). Our best-fit model for the solar proxy spectrum of Vesta, shown in Figure 5, yields an abundance of $A(\mathrm{Be})_{\odot}=1.30$ dex. The location of the tuned lines are marked as in Figure 4. There is a velocity offset between the observed and modeled profiles of the blended $\mathrm{Be}$ feature of $\sim 0.6 \mathrm{~km} \mathrm{~s}^{-1}$. Models adopting the previously

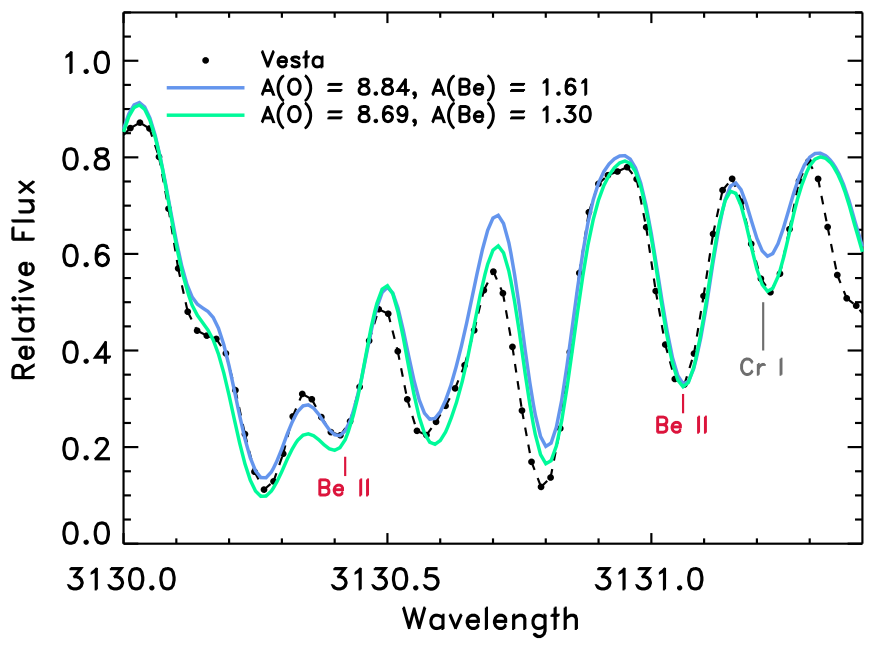

Figure 6. Solar spectrum from an observation of Vesta (dashed line) compared to synthetic spectra generated adopting a "high" oxygen abundance for the Sun (blue line) and for adopting the "low" abundance as in Figure 5 (green line). In both cases, the Be abundance was adjusted to provide the best fit to the Be line at $\lambda 3131.065 \AA$. The previously tuned $\mathrm{Cr}$ line is indicated.

accepted "depleted" (1.15 dex) and "undepleted" (1.38 dex) $A$ (Be) are also shown. Assuming the continuous opacity is correctly characterized, then the uncertainty associated with this measurement is estimated to be $\sim 0.1$ dex. Our measurement is therefore consistent with undepleted Be in the solar photosphere within the uncertainties, whether the slightly higher $(A(\mathrm{Be})=1.38 \mathrm{dex}$; Asplund et al. 2009) or lower $(A(B e)=1.32$ dex; Lodders 2010) meteoritic abundance is adopted. Taken at face value, our measurement suggests at most a modest $5 \%-20 \%$ depletion in Be.

However, as we noted in Section 1, there is an unresolved controversy on the solar oxygen abundance, where recent photospheric measurements from spectroscopy utilizing the latest 3D NLTE stellar modeling have resulted in a decrease in the accepted solar oxygen abundance (Caffau et al. 2015 and Asplund et al. 2009.) The solar oxygen abundance adopted in this work is "low," at $A(\mathrm{O})_{\odot}=8.69$ dex. In the past, much higher $A(\mathrm{O})$ have been accepted (e.g., $A(\mathrm{O})_{\odot}=8.93$ dex by Anders 1989), and some more recent studies still find "high" abundances (e.g., 8.81 dex by Caffau et al. 2008 and 8.86 dex by Centeno \& Socas-Navarro 2008). The larger $\mathrm{O}$ abundances provide better agreement with helioseismology (see, e.g., Guzik et al. 2006).

To test the impact of changing the adopted solar oxygen abundance on our solar $A(\mathrm{Be})$ measurement, we rerun the opacity test of Section 3 after increasing $A(\mathrm{O})$ by 0.15 dex to 8.84 dex and re-derive Be using the new opacity factor. At this higher oxygen abundance, the model lines are deeper than in Figure 2 and we must adopt an opacity factor of $a=1.35$ to fit the observations. The resulting $A(\mathrm{Be})$ at this larger opacity is 1.61 dex, which is enhanced over the meteoritic abundance by a factor of 1.7. Figure 6 compares this new best fit with the result previously shown in Figure 5. Note that the $\mathrm{Cr}$ I line at $\lambda 3131.212 \AA$, which was already tuned by increasing the $\log g f$ would require an additional $\log g f$ increase to fit the line. Although the fit to the spectrum near the bluer, more blended feature at $\lambda 3130.422 \AA$ appears better in the "high O" case, the overall fit to the observed spectrum over the wavelength region shown in Figure 6 is better for the "low O" case, even when excluding the $\mathrm{Cr}$ line that was tuned to match the spectrum in the "low O" case. Furthermore, a Be enhancement in the solar 
Table 4

Effects of Modeling Assumptions on Solar A(Be)

\begin{tabular}{lcc}
\hline \hline Opacity Factor & Line List & $\begin{array}{c}A(\mathrm{Be}) \\
\operatorname{dex}\end{array}$ \\
\hline 0.85 & this work & 1.30 \\
0.00 & this work & 1.11 \\
0.85 & DM12 & 1.33 \\
0.00 & DM12 & 1.17 \\
0.85 & T11 & 1.30 \\
0.00 & T11 & 1.10 \\
\hline
\end{tabular}

photosphere over the meteoritic abundance would be difficult to explain physically. We therefore conclude that the lower $A$ (O) adopted here is the correct one.

Finally, we look at how the varying model assumptions (adopted opacity and components of the blend with the Be II line) influences the $A(\mathrm{Be})$ measurement by fitting the $3131.065 \AA$ feature with different combinations of continuous opacity and line lists. In all cases, the continuum placement, broadening of the spectrum, etc. all remained fixed. Only $A(\mathrm{Be})$ was varied. The results of this analysis are given in Table 4 . Note that the abundances derived using our new line list are nearly identical to those using the line list of DM12. This is because both use the tuned Mn I $\log g f$, and the equivalent width of the hypothesized Ti II line has an equivalent width of only $\sim 9.4 \mathrm{m \AA}$ in the solar spectrum. Using the line list by T11, which does not tune the Mn I but instead adopts a hypothetical Fe I, yields results in very good agreement with our results. Note however, that T11, who did not apply any adjustment to the continuous opacity, found a solar value of $A(\mathrm{Be})=1.22 \mathrm{dex}$, nearly exactly intermediate to the results obtained with the two different assumptions on the continuous opacity in this work using their line list.

\section{Summary}

We have compiled a new line list for measuring beryllium abundances in stars and have updated the MOOG stellar synthesis code to include new sources of continuous opacity. Despite the advances in the physics in the literature that we have included in our stellar models, we still require opacity and line parameter tuning that has been utilized in the past, which limits the attainable precision in the absolute scaling of the measured $A$ (Be). The treatment of the continuous opacity in particular has a profound effect on the derived abundances. For the Sun, the opacity is inter-related with the question of the photospheric oxygen abundance. Our best results favor the interpretation that the photospheric solar $A(\mathrm{Be})$ shows little depletion (a factor of 1.2 reduction taken at face value), though the results are consistent with no depletion given the uncertainties.

The authors thank Jeff Valenti for helpful discussion of UV opacity sources and to Philip Stancil for providing NH opacity data in advance of publication. We also thank the referee for his or her careful reading of our manuscript and thoughtful suggestions that improved the presentation of our results.

This work has made use of the VALD database, operated at Uppsala University, the Institute of Astronomy RAS in Moscow, and the University of Vienna.

Based, in part, on data collected at Subaru Telescope and obtained from the SMOKA, which is operated by the Astronomy Data Center, National Astronomical Observatory of Japan."
Facilities: Subaru (HDS), HST (STIS).

Software: MOOG (Sneden 1973), IRAF (Tody 1986, 1993).

\section{ORCID iDs}

Joleen K. Carlberg (ib https://orcid.org/0000-0001-5926-4471

\section{References}

Allende Prieto, C., \& Lambert, D. L. 2000, AJ, 119, 2445

Anders, E. 1989, GeCoA, 53, 197

Asplund, M. 2004, A\&A, 417, 769

Asplund, M. 2005, AR\&A, 43, 481

Asplund, M., Grevesse, N., Sauval, A. J., \& Scott, P. 2009, ARA\&A, 47, 481

Ayres, T. R. 2010, ApJS, 187, 149

Ayres, T. R. 2013, AN, 334, 105

Baba, H., Yasuda, N., Ichikawa, S.-I., et al. 2002, in ASP Conf. Ser. 281, Astronomical Data Analysis Software and Systems XI, ed. D. A. Bohlender et al. (San Francisco, CA: ASP), 298

Balachandran, S. C., \& Bell, R. A. 1998, Natur, 392, 791

Bell, R. A., Balachandran, S. C., \& Bautista, M. 2001, ApJL, 546, L65

Bell, R. A., Paltoglou, G., \& Tripicco, M. J. 1994, MNRAS, 268, 771

Bergemann, M., \& Gehren, T. 2008, A\&A, 492, 823

Boesgaard, A. M., \& King, J. R. 2002, ApJ, 565, 587

Caffau, E., Ludwig, H.-G., Steffen, M., et al. 2008, A\&A, 488, 1031

Caffau, E., Ludwig, H.-G., Steffen, M., et al. 2015, A\&A, 579, A88

Carlberg, J. K. 2018, Beryllium synthesis with MOOG, v1.0.0, Zenodo, doi:10 5281 /zenodo. 1306725

Carlberg, J. K., Cunha, K., Smith, V. V., \& Majewski, S. R. 2012, ApJ, 757, 109

Castelli, F., \& Kurucz, R. L. 2004, A\&A, 419, 725

Centeno, R., \& Socas-Navarro, H. 2008, ApJL, 682, L61

Chmielewski, Y., Brault, J. W., \& Mueller, E. A. 1975, A\&A, 42, 37

Cunha, K., \& Smith, V. V. 1999, ApJ, 512, 1006

Delgado Mena, E., Israelian, G., González Hernández, J. I., Santos, N. C., \& Rebolo, R. 2012, ApJ, 746, 47

Fontenla, J. M., Stancil, P. C., \& Landi, E. 2015, ApJ, 809, 157

Garcia Lopez, R. J., Severino, G., \& Gomez, M. T. 1995, A\&A, 297, 787

Grevesse, N., Asplund, M., \& Sauval, A. J. 2007, SSRv, 130, 105

Grevesse, N., \& Sauval, A. J. 1998, SSRv, 85, 161

Gustafsson, B., Edvardsson, B., Eriksson, K., et al. 2008, A\&A, 486, 951

Guzik, J. A., Watson, L. S., \& Cox, A. N. 2006, MmSAI, 77, 389

Hatzes, A. P., Cochran, W. D., Endl, M., et al. 2006, A\&A, 457, 335

Hinkle, K., Wallace, L., Valenti, J. A., \& Ayres, T. 2005, Ultraviolet Atlas of the Arcturus Spectrum, 1150-3800 A (San Francisco, CA: ASP)

Hinkle, K., Wallace, L., Valenti, J. A., \& Harmer, D. 2000, Visible and Near Infrared Atlas of the Arcturus Spectrum 3727-9300 A (San Francisco, CA: ASP)

Kupka, F., Piskunov, N., Ryabchikova, T. A., Stempels, H. C., \& Weiss, W. W. 1999, A\&AS, 138, 119

Kurucz, R. L. 1992, RMxAA, 23, 181

Kurucz, R. L., van Dishoeck, E. F., \& Tarafdar, S. P. 1987, ApJ, 322, 992

Lacour, S., Meimon, S., Thiébaut, E., et al. 2008, A\&A, 485, 561

Lodders, K. 2003, ApJ, 591, 1220

Lodders, K. 2010, Principles and Perspectives in Cosmochemistry, Vol. 16 (Berlin: Springer)

Luck, R. E., \& Heiter, U. 2007, AJ, 133, 2464

Masseron, T., Plez, B., van Eck, S., et al. 2014, A\&A, 571, A47

Melo, C. H. F., de Laverny, P., Santos, N. C., et al. 2005, A\&A, 439, 227

Peterson, R. C., \& Kurucz, R. L. 2015, ApJS, 216, 1

Ramírez, I., \& Allende Prieto, C. 2011, ApJ, 743, 135

Richichi, A., Percheron, I., \& Davis, J. 2009, MNRAS, 399, 399

Short, C. I., \& Hauschildt, P. H. 2005, ApJ, 618, 926

Short, C. I., \& Hauschildt, P. H. 2009, ApJ, 691, 1634

Smiljanic, R., Pasquini, L., Bonifacio, P., et al. 2009, A\&A, 499, 103

Smith, V. V., Suntzeff, N. B., Cunha, K., et al. 2000, AJ, 119, 1239

Sneden, C. 1973, ApJ, 184, 839

Sneden, C., Lucatello, S., Ram, R. S., Brooke, J. S. A., \& Bernath, P. 2014, ApJS, 214, 26

Tajitsu, A., Aoki, W., Kawanomoto, S., \& Narita, N. 2010, PNAOJ, 13, 1

Takeda, Y., Tajitsu, A., Honda, S., et al. 2011, PASJ, 63, 697

Tody, D. 1986, Proc. SPIE, 627, 733

Tody, D. 1993, in ASP Conf. Ser. 52, Astronomical Data Analysis Software and Systems II, ed. R. J. Hanisch, R. J. V. Brissenden, \& J. Barnes (San Francisco, CA: ASP), 173 